University of South Carolina

Scholar Commons

$10-24-2013$

\title{
Enhanced Reversibility and Durability of a Solid Oxide Fe-Air Redox Battery by Carbothermic Reaction Derived Energy Storage Materials
}

\author{
Xuan Zhao \\ Xue Li \\ Yunhui Gong \\ Kevin Huang \\ University of South Carolina - Columbia, huang46@cec.sc.edu
}

Follow this and additional works at: https://scholarcommons.sc.edu/emec_facpub

Part of the Mechanical Engineering Commons

\section{Publication Info}

Published in Chemical Communications, Volume 50, Issue 5, 2013, pages 623-625.

(C) Chemical Communications (2013), Royal Society of Chemistry.

This article cannot be redistributed of further made available.

This article was first published by the Royal Society of Chemistry and can be found at http://dx.doi.org/ $10.1039 / \mathrm{c} 3 \mathrm{cc} 47673 \mathrm{a}$

Zhao, X., Li, X., Gong, Y. \& Huang, K. (2013). Enhanced Reversibility and Durability of a Solid Oxide Fe-Air Redox Battery by Carbothermic Reaction Derived Energy Storage Materials. Chemical Communications, 50 (5), 623 - 625. http://dx.doi.org/10.1039/c3cc47673a 


\section{ChemComm}

\section{Enhanced reversibility and durability of a solid oxide Fe-air redox battery by carbothermic reaction derived energy storage materials $\dagger$}

50,623

Received 6th October 2013,

Accepted 22nd October 2013

DOI: $10.1039 /$ c3cc47673a

www.rsc.org/chemcomm

\author{
Xuan Zhao, Xue Li, Yunhui Gong and Kevin Huang*
}

The recently developed solid oxide metal-air redox battery is a new technology capable of high-rate chemistry. Here we report that the performance, reversibility and stability of a solid oxide iron-air redox battery can be significantly improved by nanostructuring energy storage materials from a carbothermic reaction.

For the last twenty years, the problems of stability and safety occurring at high rates have been one of the greatest challenges for lithium-ion batteries to progress from portable consumer electronics to mid-scale all-electric/hybrid vehicles and largescale grid storage. With the existing material systems and engineering design, it would seem formidable to overcome this grand challenge in years to come. The discovery of new functional materials, identification of new storage mechanisms and computational material design are deemed imperative to realize the next generation of advanced high-rate lithium-ion and other rechargeable batteries.

Recently, we have made a significant effort to identify new mechanisms for advanced energy storage; this effort has culminated in demonstrating a new "metal-air" chemistry that utilizes solid oxide-ion conductors as an electrolyte. ${ }^{1-9}$ The new battery, termed a solid oxide metal-air redox battery or SOMARB, comprises two key functional components: a reversible solid oxide fuel cell (RSOFC) and a metal/metal oxide redox couple. The former functions as the electrical unit to realize the charge and discharge cycles by utilizing the electrolysis and fuel cell modes, respectively, while the latter acts as the energy storage medium (ESM) located next to, but not in contact with, the fuel-electrode of the RSOFC to store the associated chemical energy in situ. An innovative oxygen shuttle $\mathrm{H}_{2}-\mathrm{H}_{2} \mathrm{O}$ is enclosed between the RSOFC and the ESM to promote the energy conversion.

Department of Mechanical Engineering, University of South Carolina, Columbia, SC 29201, USA. E-mail: kevin.huang@sc.edu; Fax: +1 803-777-0106; Tel: +1 803-777-4185

$\dagger$ Electronic supplementary information (ESI) available. See DOI: 10.1039/c3cc47673a
A number of important advantages are noted for the SOMARB when compared to conventional rechargeable metal-air batteries. First, the reactant and product at the oxygen electrode of the SOMARB are gaseous $\mathrm{O}_{2}$, avoiding physically clogging the air pathway as condensed phases which form in other metal-air batteries with cation conducting electrolytes. Second, a solid/ solid interface in the SOMARB is chemically more stable than its solid/liquid counterpart, drastically reducing the possibility of invoking detrimental chemical reactions which could cause performance decay, rate-capacity limitations and safety problems. Third, the SOMARB is capable of operating at high rates since the rate-limiting volume-changes induced by oxidationreduction in the ESM do not affect the electrical functionality of a RSOFC due to a decoupled RSOFC-ESM design. ${ }^{1-9}$

The functionality of the SOMARB as a storage battery has been compellingly demonstrated in our laboratory by the chemistry of iron-air, ${ }^{1,2,5-8}$ tungsten-air ${ }^{3}$ and molybdenum-air. ${ }^{4}$ As the model system, the iron-air battery has also been thoroughly studied in the perspective of energy capacity, reversibility (round-trip efficiency), current density (rate capacity), state of charge (metal utilization) and durability (cycle life) over a broad temperature range (550-800 $\left.{ }^{\circ} \mathrm{C}\right) .^{1,2,5-8}$ From a computational perspective, a multi-physics-based model has recently been constructed and satisfactorily verified with the experimental results obtained under high current density. ${ }^{9}$ Similar high temperature metalair batteries have also been reported by other groups. ${ }^{10-17}$ Improvements in the RSOFC and ESM have been made on the rate capacity and redox kinetics. ${ }^{10-12,15,17} \mathrm{Mg}$-air chemistry was reported as a variation of the new metal-air battery, although it is non-rechargeable. ${ }^{16}$ There was also interest from industry in commercializing this type of battery for grid storage in the future. ${ }^{13,14}$ Overall, it is clear that the SOMARB is capable of achieving high energy capacity at high rate capacity.

While the SOMARB's potential as a high rate-capacity battery has been consistently demonstrated in these studies, its reversibility and durability have not yet been fully established for commercial applicability. Both the performances of the RSOFC 
and the ESM are understood to play a role in the SOMARB's reversibility and durability. In fact, the RSOFC has been previously identified as a source of degradation observed in the SOMARB. ${ }^{6,8}$ Therefore, maintaining the reversibility and durability of the RSOFC for repeated operations of electrolysis as a fuel cell is of great importance to the success of the SOMARB technology.

In this study, we report that the properties of an ESM can also affect the SOMARB's reversibility and durability. We select the Fe-based ESM as the model system, but have synthesized it by a different method: an iron carbothermic reaction. A detailed description of the carbothermic synthesis as well as thermodynamic analysis of the reaction is given in the ESI. $\dagger$ Since CO and $\mathrm{CO}_{2}$ are products of the reaction, their departure from solid surfaces can create a unique porous nanostructure with a very high surface area; this microstructural feature is deemed very important to achieve a fast redox reaction. The initial metallic Fe also eliminates a reduction step in the battery operation and allows for a greater loading of capacity-determining active metals than oxide precursor. The excess of carbon present in the original ESM could also act as an active fuel to engage in the redox reaction for additional storage capacity. To distinguish from the conventional Fe-based ESM, we use Fe/C to describe the ESM derived from the carbothermic reaction in this study.

SEM images illustrating microstructures of the as synthesized Fe/C ESM are shown in Fig. 1(a). The particles are clearly uniform with a size of $\sim 100 \mathrm{~nm}$. EDS analysis reveals that the bulk (zone-1 and -2) as well as the surface (zone-3) contain the elements $\mathrm{Fe}, \mathrm{C}, \mathrm{Zr}$ and $\mathrm{O}$, but with the surface exhibiting a slightly higher $\mathrm{C}$ content. It appears that a "core-shell" (zone-2) structure has formed after the reaction. As indicated in Fig. S4 of ESI, $\dagger$ the Fe/C ESM's morphology did not change much even after the long-term testing. We could not study the internal "core-shell" structure further with TEM, however, owing to concerns about potential damage to the lens due to the magnetism present in the iron particles.

The Ellingham diagram shown in Fig. S1 of ESI $\dagger$ suggests that a reaction temperature of $1000{ }^{\circ} \mathrm{C}$ is sufficient to drive the
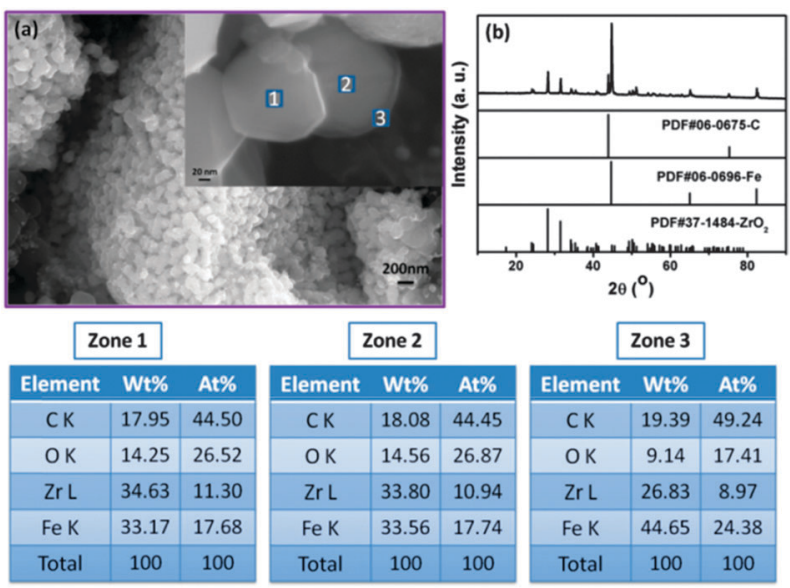

Fig. 1 Microstructure and composition of the Fe/C ESM obtained by an iron carbothermic reaction; (a) morphology and (b) XRD pattern. iron carbothermic reaction to completion. The XRD patterns shown in Fig. 1(b) are also consistent with the EDS finding, i.e., the primary phases in the as-synthesized ESM are $\mathrm{Fe}, \mathrm{ZrO}_{2}$ and C. The BET analysis yields a surface area of $12.5 \mathrm{~m}^{2} \mathrm{~g}^{-1}$ for the as-synthesized powder, which is more than four times greater than the conventional Fe ESM. ${ }^{5}$ These microstructural features are excellent attributes for a reversible redox couple.

The basic energy storage characteristics of the Fe/C-air battery evaluated over a 10-cycle period of our standard testing protocol at $550{ }^{\circ} \mathrm{C}$ are shown in Fig. 2. The battery was continuously cycled at a current density $j=10 \mathrm{~mA} \mathrm{~cm}{ }^{-2}$ for ten consecutive 10 min cycles, producing a constant discharge specific energy (DSE) of $1258 \mathrm{~W} \mathrm{~h}$ per $\mathrm{kg}$ of Fe, which is $93 \%$ of the maximum theoretical specific energy (MTSE) of $1360 \mathrm{~W}$ h per $\mathrm{kg}$ of Fe. The round-trip efficiency (RTE) is $83.3 \%$ when the DSE is compared with a charge specific energy (CSE) of $1510 \mathrm{~W} \mathrm{~h}$ per $\mathrm{kg}$ of Fe. This performance is even better than the optimized $550{ }^{\circ} \mathrm{C}$ Fe-air battery previously reported. ${ }^{5}$ No degradation is detectable during the cycle period. It should be noted that the specific energy shown in Fig. 2 and the following figures are all normalized to the mass of Fe actually consumed by the oxygen flux provided by the RSOFC. Such a normalization allows us to determine the degree of energy loss and compare with results previously published. Also noted from Fig. 2 is that the battery was operated at a relatively low power density. Clearly, better performance from the RSOFC is desirable for low temperature operation.

The long-term cyclic stability of the $\mathrm{Fe} / \mathrm{C}$-air battery under $j=10 \mathrm{~mA} \mathrm{~cm}{ }^{-2}$ is shown in Fig. 3, and it is further compared with the baseline Fe-air battery at $550{ }^{\circ} \mathrm{C}$ for the same 100 cycles. The baseline ESM refers to that derived from a co-precipitation previously reported. ${ }^{1-9}$ Evidently, the Fe/C-air battery shows a superior stability over the baseline. The average degradation rate for the $\mathrm{Fe} / \mathrm{C}$-air battery is estimated to be $214 \%$ lower than the baseline. The DSE and RTE of the Fe/C-air battery averaged

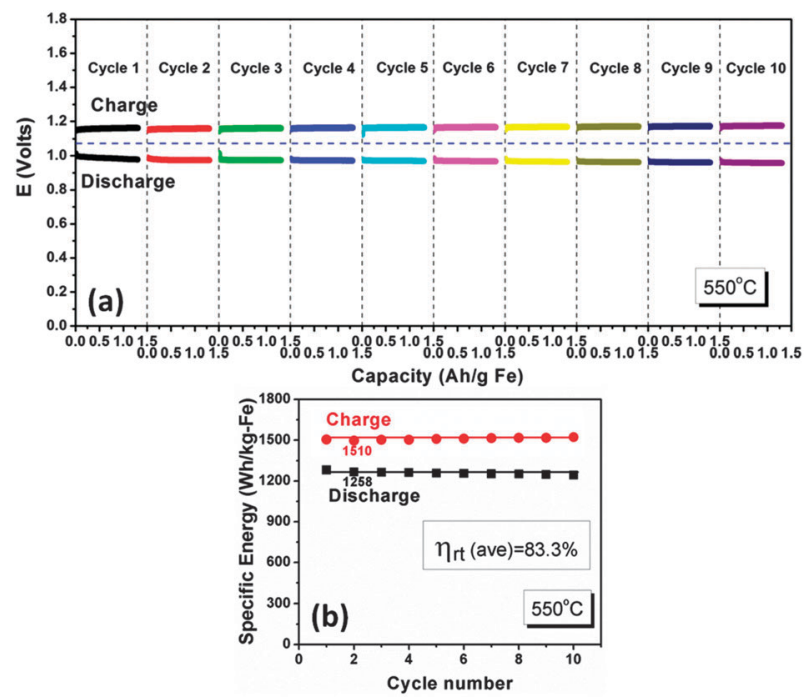

Fig. 2 Cyclic performance of the Fe/C-air battery operated at $550{ }^{\circ} \mathrm{C}$ under a current density of $10 \mathrm{~mA} \mathrm{~cm}^{-2}$ (a) $E$ vs. charge capacity; (b) average specific energy vs. number of cycles. 


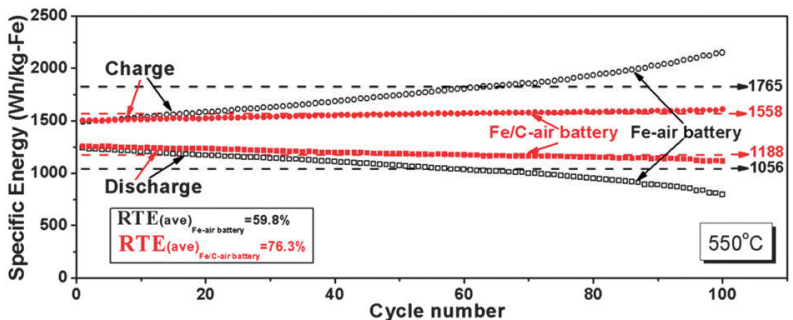

Fig. 3 Plot of DSE/CSE as a function of the number of cycles under $550{ }^{\circ} \mathrm{C}$ and $j=10 \mathrm{~mA} \mathrm{~cm}^{-2}$.

from the 100 cycles are $1188 \mathrm{~W}$ h per $\mathrm{kg}$ of $\mathrm{Fe}$ and $76.3 \%$, respectively; this level of performance represents a $12.5 \%$ and 27.6\% improvement over the baseline (1188vs. 1056 and 76.3\% vs. $59.8 \%) .{ }^{5}$ The lower DSE and RTE for the 100-cycle test relative to the initial 10-cycle study shown in Fig. 3 (1188vs. $1258 \mathrm{~W}$ h per $\mathrm{kg}$ of Fe and $76.3 \%$ vs. 83.3\%) reflect the degree of loss in energy and reversibility throughout the 100-cycle period. The electrochemical impedance spectra shown in Fig. S3 of the $\mathrm{ESI} \dagger$ further confirm that the resistance of the $\mathrm{Fe} / \mathrm{C}$-air battery is consistently lower than the baseline after the test. A general degradation mechanism for the SOMARB has been previously identified and discussed. ${ }^{2}$

The voltage as a function of state-of-charge was also investigated. The state-of-charge of the SOMARB is represented by the utilization of metal (discharge) or metal-oxide (charge) in the ESM. To show a wider range of state-of-charge, a longer cycle duration is needed to have an appreciable amount of metal or metal oxide utilized. Since the SOMARB exhibits an EMF fixed by the thermodynamic equilibrium between the metal and metal-oxide, ${ }^{1,2,5}$ its operating voltage should be independent of the state-of-charge under a galvanic cycle until all the active metals or metal-oxides are depleted. In other words, the amount of metal loading essentially determines the storage capacity of a SOMARB.

A single-cycle plot of voltage $v s$. metal/metal-oxide utilization measured at $550{ }^{\circ} \mathrm{C}$ is shown in Fig. 4. The battery was cycled between $0.80 \mathrm{~V}$ and $1.80 \mathrm{~V}$ under a constant $j=10 \mathrm{~mA} \mathrm{~cm}{ }^{-2}$.

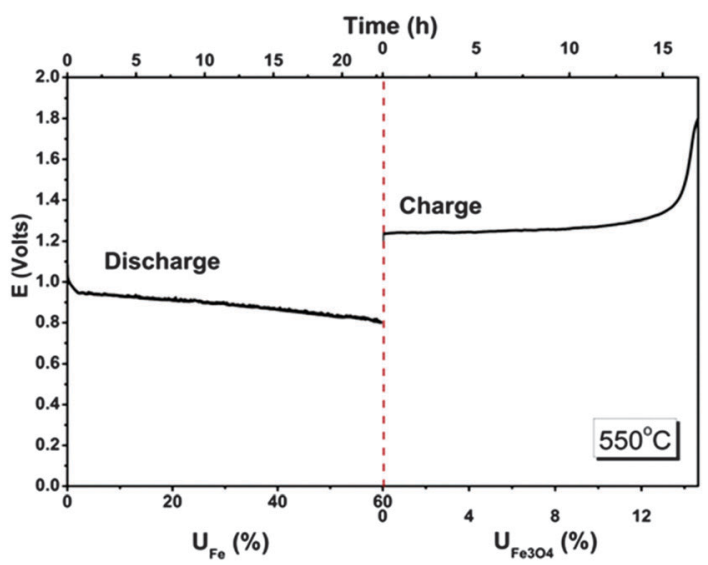

Fig. 4 The effect of state-of-charge (metal/metal-oxide utilization) on the SOMARB's terminal voltage.
The total discharge time reached $23 \mathrm{~h}$ during which $60 \%$ of $\mathrm{Fe}$ was utilized with a relatively stable voltage. Approximately $15 \mathrm{~h}$ were needed for the charge cycle to reach $\sim 13 \%$ utilization of $\mathrm{Fe}_{3} \mathrm{O}_{4}$ with a stable voltage prior to an abrupt increase in the charging voltage, a sign of $\mathrm{Fe}_{3} \mathrm{O}_{4}-\mathrm{H}_{2} \mathrm{O}$ depletion. The battery produced a DSE $=1132 \mathrm{~W}$ h per $\mathrm{kg}$ of $\mathrm{Fe}$ at a RTE $=93.7 \%$ when compared with a CSE $=1208 \mathrm{~W}$ h per $\mathrm{kg}$ of Fe. Overall, the battery's terminal voltage exhibits near independence from the state-of-charge. The asymmetrical discharge-charge curve in Fig. 4 implies that the reduction of $\mathrm{Fe}_{3} \mathrm{O}_{4}$ and/or $\mathrm{H}_{2} \mathrm{O}$ could be a slower process than the oxidation of $\mathrm{Fe}$ and/or $\mathrm{H}_{2} \mathrm{O}$ production. This could be partially related to a "concentration" effect since the ratio of equilibrium partial pressures $\mathrm{H}_{2}$ to $\mathrm{H}_{2} \mathrm{O}$ is $79 / 21$ (equivalent to the $\mathrm{OCV}$ condition) at $550{ }^{\circ} \mathrm{C}$, a condition which is more favourable to discharge than charge.

In summary, the conventional iron carbothermic reaction was investigated in this study as a means of producing a high surface-area nanostructured ESM. The electrochemical characterization of a SOMARB using the Fe/C ESM shows an improvement in DSE and RTE by $12.5 \%$ and $27.8 \%$, respectively, over the baseline performance. The most pronounced improvement from the new Fe/C ESM is the long-term cyclic stability, averaging $214 \%$ better than the baseline performance. A long-duration cyclic study further demonstrates a near state-of-charge independent terminal voltage, a desirable attribute for any type of electrochemical battery. Overall, the carbothermic reaction proves itself to be a viable approach to making a high surface-area, low-cost and stable ESM for high capacity and durable SOMARBs.

\section{Notes and references}

1 N. Xu, X. Li, X. Zhao, J. B. Goodenough and K. Huang, Energy Environ. Sci., 2011, 4, 4942-4946.

2 X. Zhao, N. Xu, X. Li, Y. Gong and K. Huang, RSC Adv., 2012, 2, 10163-10166.

3 X. Zhao, X. Li, Y. Gong, N. Xu, K. Romito and K. Huang, Chem. Commun., 2013, 49, 5357-5359.

4 X. Zhao, Y. Gong, X. Li, N. Xu and K. Huang, J. Mater. Chem. A, 2013, 1, 14858-14861.

5 X. Zhao, Y. Gong, X. Li, N. Xu and K. Huang, J. Electrochem. Soc., 2013, 160, A1241-A1247.

6 X. Zhao, N. Xu, X. Li, Y. i. Gong and K. Huang, ECS Trans., 2013, 45, 113-121.

7 X. Zhao, N. Xu, X. Li, Y. Gong and K. Huang, ECS Trans., 2013, 50, $115-123$.

8 X. Zhao, Y. Gong, X. Li, N. Xu and K. Huang, J. Electrochem. Soc., 2013, 160, A1716-A1719.

9 M. Guo, X. Zhao, R. E. White and K. Huang, J. Electrochem. Soc., 2013, 160, A2085-A2092.

10 A. Inoishi, S. Ida, S. Uratani, T. Okano and T. Ishihara, Phys. Chem. Chem. Phys., 2012, 14, 12818-12822.

11 A. Inoishi, Y. W. Ju, S. Ida and T. Ishihara, J. Power Sources, 2013, 229, 12-15.

12 A. Inoishi, S. Ida, S. Uratani, T. Okano and T. Ishihara, RSC Adv., 2013, 3, 3024-3030.

13 W. Drenckhahn, H. Greiner, M. Kuhne, H. Landes, A. Leonide, K. Litzinger, C. Lu, C. Schuh, J. Shull and T. Soller, ECS Trans., 2013, 50, 125-135.

14 H. Landes and R. Reichenbacher, ECS Trans., 2013, 50, 47-68.

15 A. Inoishi, Y. Okamoto, Y. W. Ju, S. Ida and T. Ishihara, RSC Adv., 2013, 3, 8820-8825.

16 A. Inoishi, Y. W. Ju, S. Ida and T. Ishihara, Chem. Commun., 2013, 49, 4691-4693.

17 H. Ohmori, S. Uratani and H. Iwai, J. Power Sources, 2012, 208, 383-390. 